\title{
ORIGINAL
}

\section{Lung ultrasound predicts clinical course and outcomes in COVID-19 patients}

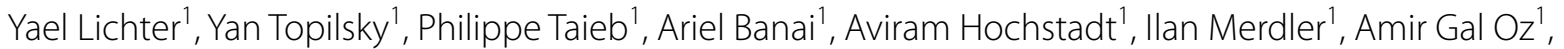
Jacob Vine1, Or Goren ${ }^{1}$, Barak Cohen ${ }^{1}$, Orly Sapir ${ }^{1}$, Yoav Granot ${ }^{1}$, Tomer Mann', Shirley Friedman ${ }^{1}$, Yoel Angel ${ }^{1}$, Nimrod Adi ${ }^{1}$, Michal Laufer-Perl ${ }^{1}$, Merav Ingbir ${ }^{1}$, Yaron Arbel ${ }^{1}$, Idit Matot ${ }^{1}$ and Yishay Szekely ${ }^{1,2^{*}}$ (D)

(c) Springer-Verlag GmbH Germany, part of Springer Nature 2020, corrected publication 2020

\begin{abstract}
Purpose: Information regarding the use of lung ultrasound (LUS) in patients with Coronavirus disease 2019 (COVID19) is quickly accumulating, but its use for risk stratification and outcome prediction has yet to be described. We performed the first systematic and comprehensive LUS evaluation of consecutive patients hospitalized with COVID-19 infection, in order to describe LUS findings and their association with clinical course and outcome.

Methods: Between 21/03/2020 and 04/05/2020, 120 consecutive patients admitted to the Tel Aviv Medical Center due to COVID-19, underwent complete LUS within $24 \mathrm{~h}$ of admission. A second exam was performed in case of clinical deterioration. LUS score of 0 (best) - 36 (worst) was assigned to each patient. LUS findings were compared with clinical data.

Results: The median baseline total LUS score was 15, IQR [7-20]. Baseline LUS score was 0-18 in 80 (67\%) patients, and 19-36 in 40 (33\%) patients. The majority had patchy pleural thickening $(n=100 ; 83 \%)$, or patchy subpleural consolidations $(n=93 ; 78 \%)$ in at least one zone. The prevalence of pleural thickening, subpleural consolidations and the total LUS score were all correlated with severity of illness on admission. Clinical deterioration was associated with increased follow-up LUS scores $(p=0.0009)$, mostly due to loss of aeration in anterior lung segments. The optimal cutoff point for LUS score was 18 (sensitivity $=62 \%$, specificity $=74 \%$ ). Both mortality and need for invasive mechanical ventilation were increased with baseline LUS score > 18 compared to baseline LUS score $0-18$. Unadjusted hazard ratio of death for LUS score was 1.08 per point [1.02-1.16], $p=0.008$; Unadjusted hazard ratio of the composite endpoint (death or need for invasive mechanical ventilation) for LUS score was 1.12 per point [1.05-1.2], $p=0.0008$.
\end{abstract}

Conclusion: Hospitalized patients with COVID-19, at all clinical grades, present with pathological LUS findings. Baseline LUS score strongly correlates with the eventual need for invasive mechanical ventilation and is a strong predictor of mortality. Routine use of LUS may guide patients' management strategies, as well as resource allocation in case of surge capacity.

Keywords: COVID-19, Lung ultrasound, Acute respiratory distress syndrome, Risk stratification, Clinical outcomes

\footnotetext{
*Correspondence: yishays@tlvmc.gov.il

2 Department of Cardiology, Tel Aviv Medical Center, Weizmann 6,

6423919 Tel Aviv, Israel

Full author information is available at the end of the article

Yael Lichter and Yan Topilsky contributed equally to this work.
}

\section{重 Springer}




\section{Introduction}

The main manifestation of Coronavirus disease 2019 (COVID-19) is viral pneumonia, that may evolve to severe acute respiratory distress syndrome (ARDS) [1, 2]. Severe cases require intensive care treatment and prolonged mechanical ventilation, and often manifest multiorgan involvement such as hemodynamic instability, myocardial injury, renal dysfunction and coagulopathy [3]. Parameters reported to correlate with poor outcome are older age, comorbidities, high sequential organ failure assessment (SOFA) score, lymphopenia, elevated troponin and d-dimer greater than $1 \mathrm{mg} / \mathrm{L}$ [4].

Bilateral lung infiltrates on computed tomography $(\mathrm{CT})$ is the hallmark of severe disease, but can also appear in asymptomatic patients or precede respiratory symptoms by days [5]. The use of lung ultrasound (LUS) as a diagnostic tool in critically ill patients, for assessment of response to treatment as well as for follow-up, has become common practice [6-12].Moreover, its use has been recommended as standard of care [13]. Findings on LUS correlate with clinical course similar to findings on high resolution CT $[14,15]$ in various patient populations. Combining this powerful tool with bedside echocardiography allows rapid thorough assessment of cardiovascular and respiratory status of the patient and thus guidance of further treatment [16-18]. The cardiac manifestations of COVID-19 using bedside echocardiography were recently published [19]. Yet, although the outbreak of COVID-19 started months ago, systematic LUS evaluation of patients for risk stratification and management guidance has not been introduced into routine practice, perhaps because of the risk of infection spreading. To this end, we performed comprehensive LUS exams in consecutive COVID-19 hospitalized patients.

\section{Methods}

We studied 120 consecutive adult patients with COVID19 admitted to the medical ward or intensive care unit (ICU) at the Tel Aviv Medical Center, between $21 / 03 / 2020$ and $04 / 05 / 2020$. All patients had a diagnosis of COVID-19 confirmed by a positive reversetranscriptase polymerase chain reaction assay for SARS-CoV-2 in a respiratory tract sample. Demographic data, comorbid conditions, medications, physical examination, and laboratory findings were systematically recorded. Patients were risk stratified according to their COVID-19 modified early warning score (COVID-19 MEWS, Supplemental Table I) and SOFA score [20, 21]. At the beginning of the COVID-19 pandemic, we initiated a prospective program of performance of LUS on admission and on deterioration for all patients presenting with respiratory illness due to COVID-19 infection,

\section{Take-home message}

LUS provides risk stratification and prediction of outcomes in COVID-19, and may guide management strategies, triage and resource allocation during a pandemic.

using a pre-defined step-by-step protocol, as part of a routine patient care protocol. All patients underwent comprehensive LUS combined with bedside echocardiography within $24 \mathrm{~h}$ of admission. Patients who then experienced clinical deterioration underwent a repeated exam. Clinical deterioration was defined as either respiratory (acute new onset hypoxemia requiring mechanical ventilation, veno-venous extracorporeal membrane oxygenation, or both), or hemodynamic (persistent hypotension requiring vasopressors to maintain mean arterial pressure $\geq 65 \mathrm{mmHg}$ or having serum lactate level $>2 \mathrm{mmol} / \mathrm{L}$ despite adequate volume resuscitation). This is a retrospective study of the prospectively and systematically collected data on the lung ultrasound exams performed. The ethics committee of the Tel Aviv Medical Center approved the study, IRB number 0196-20-TLV.

\section{Follow-up and outcomes}

Clinical follow-up was obtained by daily review of all medical records. Outcome analysis started at time of baseline LUS exam. Endpoints studied were: all-cause mortality and composite endpoint comprised of death or new need for invasive mechanical ventilation. The data that support the findings of this study will be available from the corresponding author upon reasonable request.

\section{Lung ultrasound}

We performed LUS on all patients with COVID-19 using a six-zone method for each lung that included a scan of the anterior, antero-lateral, and postero-lateral aspects of the thorax. Examinations were performed by three cardiologists with expertise in LUS recording and interpretation using the same equipment (CX 50, Philips Medical Systems, Bothell, WA), with the same phased-array probe used for echocardiography. Each LUS lasts between 2-3 min, with the patient supine or semi-supine, omitting the need for position change during the examination. A point scoring system was employed for each region and ultrasound pattern: A-lines (normal reverberation artifacts of the pleural line that when accompanied by lung sliding correspond to normal aeration of the lung) were equal to 0 point; B-lines (hyperechoic lines vertical to the pleura line, arising from it and reaching the edge of the screen erasing A-lines, which represent reverberation artifact through edematous interlobular septa or alveoli) were divided to B1 (separated B-lines that 
correspond to moderate lung aeration loss) that was equal to 1 point, and B2 (coalescent B-lines that correspond to severe lung aeration loss) that was equal to 2 points; Lung consolidation that was equal to 3 points. Thus, an LUS score of 0 was normal, and 36 was worst [7]. Examples of the different patterns are shown in Fig. 1. We also documented the presence of pleural thickening and defined a homogenous vs. patchy pattern of each examination. Pleural thickening was qualitatively determined, indicating irregular pleural line either in cases of sub-pleural consolidations or in cases of B-lines accompanied by irregular pleural line. In accordance to present guidelines [22], the following measures were undertaken to minimize the risk of inadvertent infection: all studies were performed bedside at the designated COVID-19 wards using dedicated scanners that were tagged and set aside in each ward. Full personal protection equipment was used and LUS measurements were performed offline to reduce exposure time. Inter-observer variability for LUS score was determined by a second independent blinded and experienced observer, who measured the LUS score in 20 randomly selected patients. Inter-observer variability was assessed using the Bland-Altman method and the within-subject coefficient of variation. The within-subject coefficient of variation (calculated as the ratio of the standard deviation of the measurement difference to the mean value of all measurements) provides a scale-free, unitless estimate of variation expressed as a percentage.

\section{Statistical analysis}

Continuous normally distributed variables were presented as means \pm SD and compared using the Student's $t$ test. Normality was assessed using the Shapiro-Wilk test and visual inspection of quantile- quantile plots.
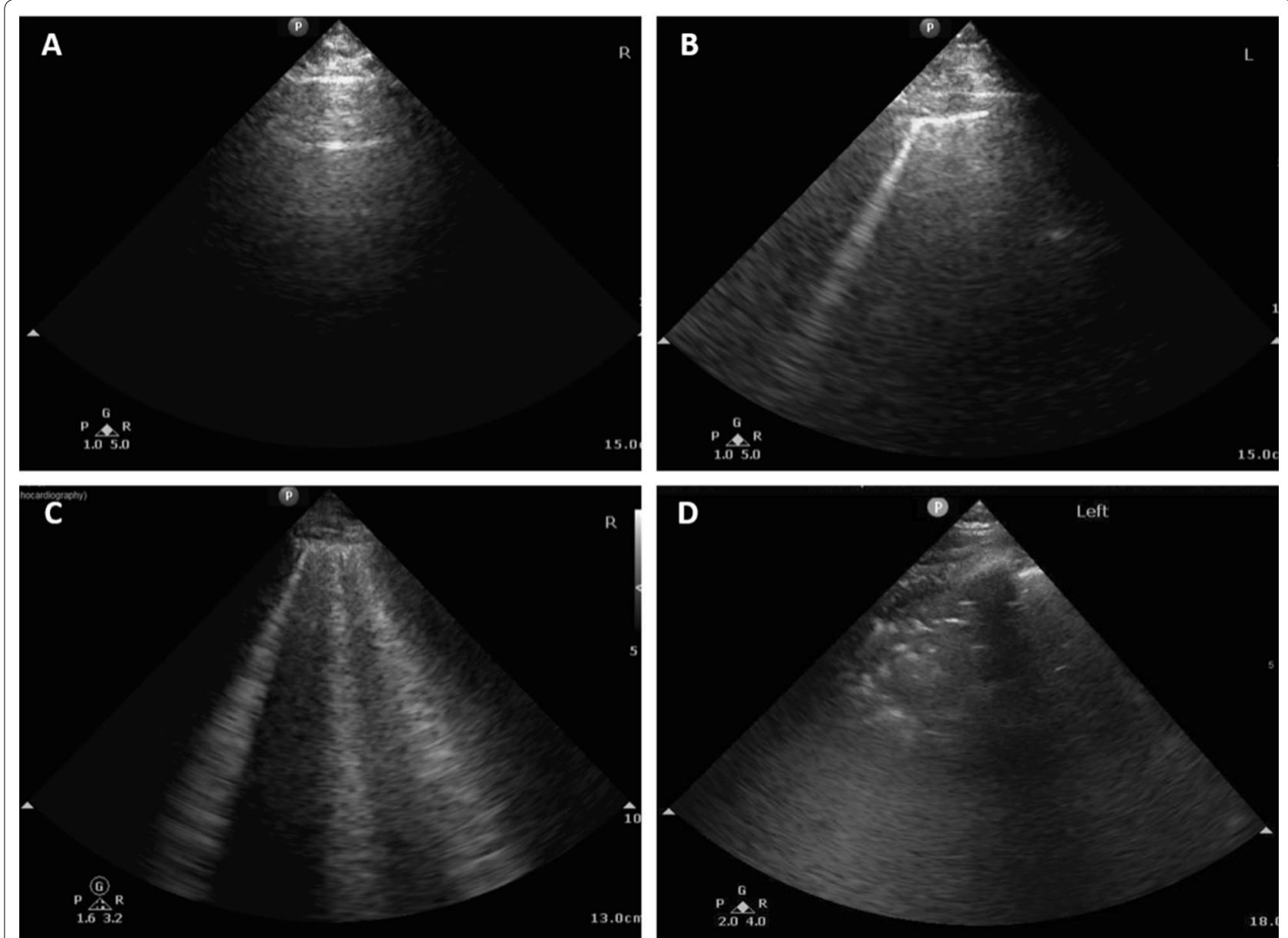

Fig. 1 Examples of different patterns of lung ultrasound findings. a A-lines, normal reverberation artifacts of the pleural line that correspond to normal aeration of the lung. $\mathbf{b}$ A single B-line that represents reverberation artifact through mildly edematous interlobular septa or alveoli that correspond to moderate aeration lost. c Multiple coalescent B-lines that correspond to severe lung aeration loss. $\mathbf{d}$ Lung consolidation that correspond to complete aeration loss 
Non-normally distributed data were presented by median, 1st and 3rd quartiles and compared using the Wilcoxon rank sum test. Categorical data were compared between groups using the $\chi^{2}$ test, or Fisher's exact test. LUS parameters in consecutive exams were compared using the signed Wilcoxon signed-rank test. Correlation between change in positive end-expiratory pressure (PEEP) and change in LUS score was examined using Spearman's rank correlation coefficient. Receiver-operating characteristic (ROC) curve analysis was used to determine optimal cutoff values of LUS score for 30-day events. The best cutoff value was defined by Youden's index calculation. Cox proportional hazards models for mortality or clinical deterioration as endpoints allowed for calculation of hazard ratios (HR) of baseline LUS parameters. $p$ values of less than 0.05 were considered to indicate statistical significance. All data were analyzed with the JMP System software version 12.0 (SAS Institute, Inc, Cary, NC). All authors participated in designing the study, collecting and analyzing data, and drafting and revising the manuscript.

\section{Results}

During the study period, clinical data were collected for 135 consecutive patients hospitalized with COVID19. Fifteen patients were excluded because they did not undergo LUS due to hospital discharge $\leq 24 \mathrm{~h}$ (8 patients), patient refusal (1 patient) and a "do not resuscitate/intubate" status in 6 patients. Thus, the study group included 120 COVID-19 patients who underwent LUS evaluation. Table 1 shows baseline characteristics and LUS assessments of all patients, stratified by LUS score tertiles. Eighty patients (67\%) had a baseline LUS score of $0-18$, and $40(33 \%)$ had an LUS score of 19-36. Mean age was $64.7 \pm 18$ years, $62 \%$ males. Comorbidities were present in $81 \%$ of patients, with hypertension being the most common, followed by diabetes and obesity. The most common symptoms on admission were respiratory, followed by only fever, chest pain and fatigue. C-reactive protein (CRP), Troponin-I, brain natriuretic peptide (BNP) and D-dimer were elevated at baseline in $88 \%$, $28 \%, 37 \%$ and $69 \%$ of patients, respectively. Patients in the upper tertile of LUS score, compared to those in the lower tertiles, were older, had lower levels of hemoglobin, lymphocytes and albumin with higher levels of CRP, troponin, D-dimer and fibrinogen $(p<0.05$ for all). They had lower ambient $\mathrm{O}_{2}$ saturation and higher SOFA score and MEWS ( $p<0.001$ for all). Baseline mean left ventricular ejection fraction was $57.7 \pm 5 \%$, mean $E / e^{\prime}$ was $10.3 \pm 6.3$ and none of the echocardiographic parameters was significantly different between the groups $(p>0.2$ for all, Supplemental Table II). Bilateral infiltrates were the most common chest X-ray manifestation, found in 39\% of patients. Pleural effusion and lobar infiltrates were rare $(<15 \%$ each).

None of the patients had normal LUS (A-lines accompanied by lung sliding in all zones), or homogenous B-lines in all zones. Most patients had patchy pleural thickening $(n=100 ; 83 \%)$, or patchy subpleural consolidations $(n=93 ; 78 \%)$ in at least one zone. Pleural effusion was rare $(n=9,8 \%)$. The median total lung score was 15, IQR [7-20]. Comparison of inter-observer variability for LUS score showed good agreement between measurements: mean difference $0.1 \pm 0.05$ points, $r=0.92$, $p=0.36$. The Bland-Altman plot showed a random scatter of points around 0 , indicating no systematic bias or measurement error proportional to the measurement value. Measurement variability (within-subject coefficient of variation) for measurements of inter-observer differences was $3.1 \%$.

\section{LUS and clinical severity grade}

On admission (baseline LUS evaluation), 75 patients were stratified as having clinically mild disease (oxygen saturation $\geq 94 \%$ at room air), 31 as moderate disease (need for non-invasive oxygen) and 14 as severe disease (need for invasive mechanical ventilation). When compared to patients with mild disease, patients with severe or moderate disease were more hypoxemic $\left(\mathrm{O}_{2}\right.$ saturation of $86 \pm 7,88.7 \pm 6 \%$ and $96.2 \pm 3 \%$ in severe, moderate and mild disease, respectively, $p<0.0001$ for trend), more tachycardic, more pyretic, required more vasopressor support and had higher levels of CRP, D-dimer and cardiac biomarkers (troponin-I, BNP). Results of LUS evaluation stratified by severity of disease are shown in Table 2. The prevalence of pleural thickening, subpleural consolidations and the total LUS score were higher with worsening disease.

\section{LUS and clinical deterioration}

In 20 patients, sequential LUS exams were performed due to clinical deterioration (hemodynamic instability $n=4$, respiratory deterioration $n=16$ ). In this group of patients, total LUS score worsened mostly due to deterioration in anterior segments grade $(16 / 20,80 \%)$ with amplification of B-lines and consolidations (Supplemental Table III). In seven patients, who were invasively ventilated during baseline LUS and underwent a repeated LUS because of further deterioration, a significant positive correlation was found between the change in LUS score and the change in PEEP requirements $(\rho=0.87$; $p=0.03)$.

Example of LUS of a patient at baseline and after clinical deterioration is shown in Supplemental Fig. 1. 
Table 1 Baseline characteristics

\begin{tabular}{|c|c|c|c|c|}
\hline Parameter & $\begin{array}{l}\text { All patients } \\
n=120\end{array}$ & $\begin{array}{l}\text { Lung ultrasound Score } 0-18 \\
n=80\end{array}$ & $\begin{array}{l}\text { Lung ultrasound Score 19-36 } \\
n=40\end{array}$ & $p$ value \\
\hline Age, mean $\pm S D$ & $64.7 \pm 18.2$ & $62 \pm 19.1$ & $70.1 \pm 15$ & 0.013 \\
\hline Male gender, $n(\%)$ & $74(61.7)$ & $47(59.8)$ & $27(67.5)$ & 0.43 \\
\hline Cause of admission, (\%) & $\begin{array}{l}\text { Respiratory (50) } \\
\text { Respiratory failure (5) } \\
\text { ECMO transfer (2) } \\
\text { CXR (1) } \\
\text { Fever (12) } \\
\text { Chest pain (9) } \\
\text { Fatigue (5) } \\
\text { Neurologic (2) } \\
\text { Gastrointestinal (3) } \\
\text { Comorbidity (3) } \\
\text { Asymptomatic (4) } \\
\text { Other (4) }\end{array}$ & $\begin{array}{l}\text { Respiratory (45) } \\
\text { Respiratory failure (1) } \\
\text { ECMO transfer (1) } \\
\text { CXR (1) } \\
\text { Fever (13) } \\
\text { Chest pain (12) } \\
\text { Fatigue (7) } \\
\text { Neurologic (0) } \\
\text { Gastrointestinal (4) } \\
\text { Comorbidity (5) } \\
\text { Asymptomatic (6) } \\
\text { Other (5) }\end{array}$ & $\begin{array}{l}\text { Respiratory (60) } \\
\text { Respiratory failure (13) } \\
\text { ECMO transfer (2) } \\
\text { CXR (0) } \\
\text { Fever (10) } \\
\text { Chest pain (2) } \\
\text { Fatigue (3) } \\
\text { Neurologic (5) } \\
\text { Gastrointestinal (2) } \\
\text { Comorbidity (0) } \\
\text { Asymptomatic (0) } \\
\text { Other (3) }\end{array}$ & 0.021 \\
\hline Body surface area, mean ( $\pm S D$ ) & $1.8 \pm 0.32$ & $1.8 \pm 0.3$ & $1.8 \pm 0.3$ & 0.73 \\
\hline \multicolumn{5}{|l|}{ Medical history } \\
\hline Ischemic heart disease, $n(\%)$ & $21(17.5)$ & $13(16.5)$ & $8(20)$ & 0.62 \\
\hline Congestive heart failure, $n(\%)$ & $11(9.2)$ & $6(7.6)$ & $5(12.5)$ & 0.50 \\
\hline S/P coronary artery bypass graft surgery, $n(\%)$ & $7(5.8)$ & $4(5.1)$ & $3(7.5)$ & 0.69 \\
\hline Atrial fibrillation/flutter, $n$ (\%) & $21(17.5)$ & $11(13.9)$ & $10(25)$ & 0.20 \\
\hline Transient ischemic attack/Stroke, $n(\%)$ & $14(11.7)$ & $9(11.4)$ & $5(12.5)$ & 0.99 \\
\hline Chronic kidney disease, $n(\%)$ & $13(10.8)$ & $6(7.6)$ & $7(17.5)$ & 0.12 \\
\hline Diabetes, $n(\%)$ & $34(28.3)$ & $21(26.6)$ & $13(32.5)$ & 0.53 \\
\hline Smoking, $n(\%)$ & $13(10.8)$ & $9(11.4)$ & $4(10)$ & 0.99 \\
\hline Hypertension, $n(\%)$ & $67(55.8)$ & $43(54.4)$ & $24(60)$ & 0.70 \\
\hline \multicolumn{5}{|l|}{ Medications } \\
\hline Angiotensin converting enzyme inhibitor, $n(\%)$ & $18(15)$ & $12(15.2)$ & $6(15)$ & 0.99 \\
\hline Angiotensin receptor blocker, $n(\%)$ & $19(15.8)$ & $11(13.9)$ & $8(20)$ & 0.43 \\
\hline Systemic corticosteroids, $n(\%)$ & $3(2.5)$ & $1(1.3)$ & $2(5)$ & 0.26 \\
\hline Other anti-inflammatories, $n(\%)$ & $4(3.3)$ & $4(5.1)$ & $0(0)$ & 0.30 \\
\hline \multicolumn{5}{|l|}{ Baseline laboratory results } \\
\hline Hemoglobin, g/dL, mean \pm SD & $13.1 \pm 2.1$ & $13.3 \pm 1.9$ & $12.5 \pm 2.4$ & 0.04 \\
\hline White blood cells, $10^{3} / \mu \mathrm{L}$, median (IQR) & $7.1(5.3-9.7)$ & $6.7(5.1-9.4)$ & $7.4(5.9-12.4)$ & 0.09 \\
\hline Neutrophils, $10^{3} / \mu \mathrm{L}$, median (IQR) & $4.7(3.4-7.4)$ & $4.3(3.2-6.9)$ & $6.1(3.8-10.6)$ & 0.015 \\
\hline Lymphocytes, $10^{3} / \mu \mathrm{L}$, median (IQR) & $1.1(0.7-1.5)$ & $1.2(0.8-1.7)$ & $0.8(0.4-1.1)$ & $<0.001$ \\
\hline Creatinine, mg/dL, median (IQR) & $0.9(0.74-1.22)$ & $0.89(0.73-1.1)$ & $0.94(0.74-1.5)$ & 0.33 \\
\hline Blood urea nitrogen, mg/dL, median (IQR) & $19(12.75-27)$ & $18(14.5-25.5)$ & $20(14.2-35.7)$ & 0.18 \\
\hline Albumin, $g / L$, mean $\pm S D$ & $38.54 \pm 5.5$ & $40.1 \pm 5.1$ & $35.5 \pm 5.2$ & $<0.001$ \\
\hline C-reactive protein, $\mathrm{mg} / \mathrm{L}$, median (IQR) & $55.4(18.2-133.1)$ & $35.3(7.5-79.1)$ & $127.3(60.2-146.3)$ & $<0.001$ \\
\hline Troponin-I, ng/L, median (IQR) & $11(2-23.5)$ & $6(3-19)$ & $19(11-40.7)$ & $<0.001$ \\
\hline Brain natriuretic peptide, pg/mL, median (IQR) & $39(16-161.25)$ & $32(11-106.5)$ & $56(25.7-249)$ & 0.07 \\
\hline D-Dimer, mg/L, median (IQR) & $0.83(0.38-1.61)$ & $0.7(0.34-1.28)$ & $1.06(0.69-2.37)$ & 0.002 \\
\hline Fibrinogen, $\mathrm{mg} / \mathrm{dL}$, mean $\pm \mathrm{SD}$ & $541.22 \pm 148.34$ & $509.63 \pm 142.82$ & $584.9 \pm 146.7$ & 0.023 \\
\hline Ferritin, ng/mL, median (IQR) & $434.4(199.68-1196.5)$ & $410.95(173.45-877.13)$ & $537.9(265.85-1650)$ & 0.13 \\
\hline \multicolumn{5}{|l|}{ Baseline physical examination } \\
\hline Heart rate, beats/minute, mean $\pm S D$ & $83.85 \pm 16.7$ & $82.5 \pm 17.1$ & $86.5 \pm 15.6$ & 0.22 \\
\hline Systolic blood pressure, $\mathrm{mmHg}$, mean $\pm \mathrm{SD}$ & $133 \pm 21.8$ & $132.6 \pm 22.1$ & $133.7 \pm 21.3$ & 0.79 \\
\hline Diastolic blood pressure, $\mathrm{mmHg}$, mean $\pm \mathrm{SD}$ & $73.76 \pm 16.4$ & $75.7 \pm 16.6$ & $69.8 \pm 16.2$ & 0.07 \\
\hline Ambient $\mathrm{O}_{2}$ saturation, $\%$, median (IQR) & $95(89-98)$ & 97 (94-99) & $89(85-95.2)$ & $<0.001$ \\
\hline Temperature, Celsius, mean \pm SD & $37.23 \pm 0.92$ & $37.1 \pm 0.8$ & $37.4 \pm 1$ & 0.09 \\
\hline Lung crepitations, $n(\%)$ & $26(22)$ & $13(17)$ & $13(33)$ & 0.05 \\
\hline
\end{tabular}


Table 1 (continued)

\begin{tabular}{|c|c|c|c|c|}
\hline Parameter & $\begin{array}{l}\text { All patients } \\
n=120\end{array}$ & $\begin{array}{l}\text { Lung ultrasound Score } 0-18 \\
n=80\end{array}$ & $\begin{array}{l}\text { Lung ultrasound Score 19-36 } \\
n=40\end{array}$ & $p$ value \\
\hline \multicolumn{5}{|l|}{ Other baseline scores } \\
\hline $\begin{array}{l}\text { Sequential organ failure assessment score, } \\
\text { median (IQR) }\end{array}$ & $1(0-2)$ & $1(0-2)$ & $2(1-5.5)$ & $<0.001$ \\
\hline Modified early warning score, median (IQR) & $4(2-7)$ & $3(1-5)$ & $7(4-12.4)$ & $<0.001$ \\
\hline \multicolumn{5}{|l|}{ Baseline chest X-ray findings } \\
\hline Lobar infiltrates, $n(\%)$ & $14(11)$ & $10(13)$ & $4(10)$ & 0.73 \\
\hline Bilateral infiltrates, $n(\%)$ & $47(39)$ & $24(30)$ & $23(58)$ & 0.003 \\
\hline Pleural effusion, $n(\%)$ & $17(14)$ & $10(13)$ & $7(18)$ & 0.52 \\
\hline Hilar congestion, $n(\%)$ & $9(8)$ & $5(6)$ & $4(10)$ & 0.44 \\
\hline \multicolumn{5}{|l|}{ Baseline lung ultrasound } \\
\hline Pleural effusion, $n(\%)$ & $9(7.5)$ & $6(7.5)$ & $3(7.5)$ & 1.00 \\
\hline Homogenous diffuse B-lines, $n$ (\%) & $0(0)$ & $0(0)$ & $0(0)$ & NA \\
\hline Pleural thickening, $n(\%)$ & $100(83.3)$ & $60(75)$ & $40(100)$ & $<0.001$ \\
\hline Subpleural consolidations, n (\%) & $93(77.5)$ & $53(66.3)$ & $40(100)$ & $<0.001$ \\
\hline Lung ultrasound score, median (IQR) & $15(7-20)$ & $9.5(5-15)$ & $22(20-24.75)$ & $<0.001$ \\
\hline
\end{tabular}

\section{Table 2 Patients stratified by clinical presentation at baseline lung ultrasound}

\begin{tabular}{|c|c|c|c|c|}
\hline \multirow[t]{2}{*}{ Parameter } & \multicolumn{3}{|c|}{ Clinical grade $^{a}$} & \multirow[t]{2}{*}{$p$ value } \\
\hline & $\begin{array}{l}\text { Mild } \\
n=75\end{array}$ & $\begin{array}{l}\text { Moderate } \\
n=31\end{array}$ & $\begin{array}{l}\text { Severe } \\
n=14\end{array}$ & \\
\hline Age, years, mean $\pm S D$ & $64.2 \pm 21$ & $72.3 \pm 13$ & $72.5 \pm 24$ & 0.12 \\
\hline Male gender, $n(\%)$ & $43(57)$ & $21(67)$ & $10(71)$ & 0.43 \\
\hline Modified early warning score, median (IQR) & $3(1-4)$ & $7(6-10)$ & $13(9-16)$ & $<0.0001$ \\
\hline Temperature, Celsius, mean \pm SD & $37.1 \pm 0.7$ & $37.4 \pm 0.8$ & $37.8 \pm 1.3$ & 0.02 \\
\hline $\mathrm{O}_{2}$ saturation, $\%$, mean $\pm \mathrm{SD}$ & $96.2 \pm 3$ & $88.7 \pm 6$ & $86.0 \pm 7$ & $<0.0001$ \\
\hline Heart Rate, beats/minute, mean \pm SD & $81.1 \pm 15$ & $85.2 \pm 17$ & $98.2 \pm 20$ & 0.005 \\
\hline Pressor requirement, $n(\%)$ & $0(0)$ & $0(0)$ & $2(13)$ & 0.02 \\
\hline C-reactive protein, $\mathrm{mg} / \mathrm{L}$, mean $\pm \mathrm{SD}$ & $49.2 \pm 45$ & $106 \pm 65$ & $162.3 \pm 68$ & $<0.0001$ \\
\hline D-dimer, mg/L, median (IQR) & $0.7(0.3-1.1)$ & $1.1(0.7-1.7)$ & $2.5(1.8-3.8)$ & $<0.0001$ \\
\hline Troponin-I, ng/L, median (IQR) & $8(4-18)$ & $14(7-31)$ & $142(19-213)$ & $<0.0001$ \\
\hline Brain natriuretic peptide, pg/mL, median (IQR) & $37(14-105)$ & $42(24-197)$ & $75(57-223)$ & 0.04 \\
\hline Lung Crepitation, $n(\%)$ & $12(16)$ & $10(32)$ & $4(28)$ & 0.22 \\
\hline \multicolumn{5}{|l|}{ Baseline chest X-ray } \\
\hline Bilateral infiltrates, $n(\%)$ & $21(28)$ & $17(55)$ & $9(64)$ & 0.004 \\
\hline Lobar infiltrates, $n(\%)$ & $6(8)$ & $6(19)$ & $2(14)$ & 0.33 \\
\hline Pleural effusion, $n(\%)$ & $6(8)$ & $7(23)$ & $4(29)$ & 0.04 \\
\hline Hilar congestion, $n(\%)$ & $2(3)$ & $6(19)$ & $1(7)$ & 0.03 \\
\hline \multicolumn{5}{|l|}{ Baseline lung ultrasound } \\
\hline Pleural effusion, $n(\%)$ & $3(4)$ & $5(16)$ & $1(7)$ & 0.11 \\
\hline Homogenous diffuse B-lines, $n(\%)$ & $0(0)$ & $0(0)$ & $0(0)$ & NS \\
\hline Pleural thickening, $n(\%)$ & $57(73)$ & $30(97)$ & $13(93)$ & 0.009 \\
\hline Subpleural consolidations, $n(\%)$ & $53(71)$ & $27(87)$ & $13(93)$ & 0.04 \\
\hline Lung ultrasound score, median (IQR) & $12(5-18)$ & $19(14-22)$ & $23(16-28)$ & $<0.0001$ \\
\hline
\end{tabular}

a At the time of baseline lung ultrasound evaluation patients were stratified to mild disease (oxygen saturation $\geq 94 \%$ at room air) in 75 , moderate disease (need for non-invasive oxygen) in 31 and severe disease (need for mechanical ventilation) in 14 


\section{LUS and survival}

There were 23 deaths during follow-up [mean follow-up period 31 days, IQR (20-40) days]. Presence of pleural effusion, pleural thickening and high total LUS score at baseline examination were each significantly associated with increased mortality (Supplemental Table IV).

The optimal cutoff point for LUS score was 18-using the highest Youden's index in the ROC analysis for 30-day mortality (AUC 0.76; sensitivity $=62 \%$, specificity $=74 \%$ ). Survival was reduced with total LUS score $>18$ vs. LUS score $\leq 18 \quad(66 \pm 20 \%$ vs. $88 \pm 11 \%$ for 30 -day survival; $\mathrm{p}=0.01$ ). Kaplan-Meier survival curve (Fig. 2a) shows lower survival with total LUS score $>18$ compared to lower LUS score. Unadjusted hazard ratio of death for total LUS score was 1.08 [1.02-1.16] per point, $p=0.008$. The unadjusted hazard ratio of death for high risk LUS score $(>18)$ was 2.65 [1.14-6.3], $p=0.02$, suggesting a 2.6-fold increase in mortality with high risk, compared to low risk, LUS score (Supplemental Table IV). The only chest X-ray finding associated with mortality was the presence of bilateral infiltrates, and its addition to the model showed that total LUS score is independently associated with mortality when accounting for chest $\mathrm{X}$-ray findings. The only physical finding associated with mortality was ambient $\mathrm{O}_{2}$ saturation. Although total LUS score remained significantly associated with mortality when adjusted for bilateral infiltrates in chest X-ray or age, its association with mortality was lost when adjusted for ambient $\mathrm{O}_{2}$ saturation and MEWS (Table 3).

\section{LUS and composite events}

Following baseline LUS, 30 composite events occurred. Presence of pleural thickening and total LUS score were significantly associated with the composite event (Supplemental Table IV).
The rate of the composite events was increased with total LUS score $>18$ vs. LUS score $\leq 18$ ( $43 \pm 9 \%$ vs. $10.6 \pm 3 \%$ for thirty days; $p=0.0004)$. Kaplan-Meier curve (Fig. 2b) shows higher rate of the composite event with total LUS score $>18$ compared to lower score. Unadjusted hazard ratio of the composite event for total LUS score was 1.12 per point [1.05-1.2], $\mathrm{p}=0.0008$. Unadjusted hazard ratio of the composite event for high risk LUS score ( $>18)$ was 4.24 [2.06-9.1], $p<0.0001$ suggesting a 4.2-fold increase in the composite event with high risk versus low risk LUS score (Supplemental Table IV).

Addition of presence of bilateral infiltrates in chest $\mathrm{X}$-ray to the model showed that total LUS score is independently associated with the composite event when accounting for chest X-ray findings. The only physical finding associated with the composite event was ambient $\mathrm{O}_{2}$ saturation. Addition of ambient $\mathrm{O}_{2}$ saturation to the model showed that total LUS score is independently associated with the composite event when accounting for ambient $\mathrm{O}_{2}$ saturation. Although total lung LUS score remained significantly associated with the composite event when adjusted for bilateral infiltrates in chest $\mathrm{X}$-ray, age or ambient $\mathrm{O}_{2}$ saturation, its association with the composite event was lost when adjusted for MEWS (Table 3).

\section{Discussion}

COVID-19 primarily affects the lungs, and pneumonia appears to be the most frequent serious manifestation of infection [1]. During the COVID-19 pandemic, LUS was sporadically used in several centers to identify disease severity, and to assist in treatment decisions [23, 24]. The results of the present study, which used a protocoled guided systematic LUS in 120 consecutive COVID-19 patients admitted to the Tel Aviv Medical Center, show
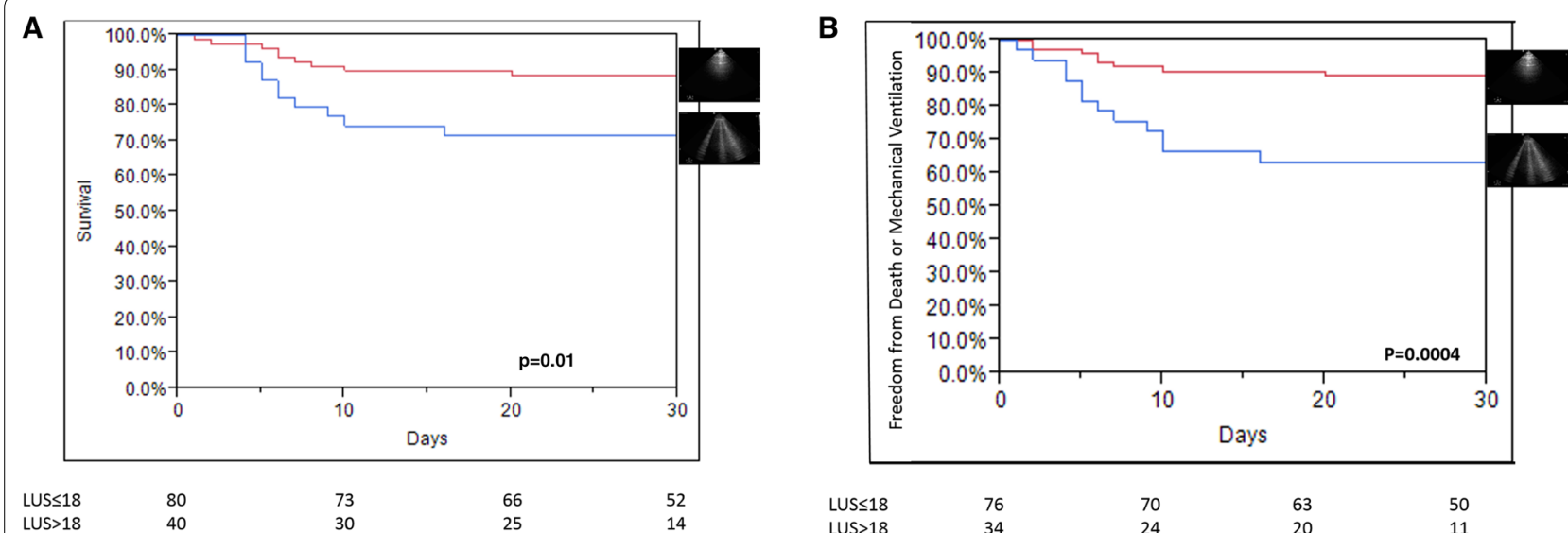

Fig. 2 a Kaplan-Meier curve for mortality according to lung ultrasound severity. b Kaplan-Meier curve for the combination of need for invasive mechanical ventilation or mortality according to lung ultrasound severity 
Table 3 Multivariable analyses of baseline predictors of clinical deterioration and death

\begin{tabular}{|c|c|c|c|}
\hline & Hazard ratio & 95\% Confidence interval & $p$ value \\
\hline \multicolumn{4}{|l|}{ Mortality } \\
\hline LUS score univariable analysis (per point) & 1.08 & $1.02-1.16$ & 0.008 \\
\hline Chest $\mathrm{X}$-ray adjustment ${ }^{\mathrm{a}}$ & 1.07 & $1.005-1.14$ & 0.03 \\
\hline Ambient $\mathrm{O}_{2}$ saturation adjustment & 1 & $0.93-1.08$ & 0.81 \\
\hline Age adjustment & 1.06 & $1.001-1.13$ & 0.05 \\
\hline Clinical adjustment $^{\mathrm{b}}$ & 0.98 & $0.92-1.05$ & 0.52 \\
\hline Age and clinical adjustment & 0.98 & $0.91-1.05$ & 0.5 \\
\hline \multicolumn{4}{|l|}{ Need for mechanical ventilation } \\
\hline LUS score univariable analysis (per point) & 1.2 & $1.1-1.3$ & $<0.001$ \\
\hline 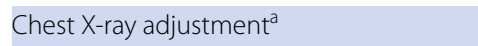 & 1.25 & $1.11-1.4$ & $<0.001$ \\
\hline Ambient $\mathrm{O}_{2}$ saturation adjustment & 1.38 & $1.14-1.76$ & $<0.001$ \\
\hline Age adjustment & 1.22 & $1.12-1.36$ & $<0.001$ \\
\hline Clinical adjustment ${ }^{b}$ & 1.19 & $1.03-1.5$ & 0.01 \\
\hline Age and clinical adjustment & 1.16 & $1.02-1.41$ & 0.02 \\
\hline \multicolumn{4}{|l|}{ Composite clinical events } \\
\hline LUS score univariable analysis (per point) & 1.12 & $1.05-1.2$ & 0.0008 \\
\hline Chest $\mathrm{X}$-ray adjustment ${ }^{\mathrm{a}}$ & 1.11 & $1.04-1.21$ & 0.002 \\
\hline Ambient $\mathrm{O}_{2}$ saturation adjustment & 1.1 & $1.03-1.2$ & 0.006 \\
\hline Age adjustment & 1.14 & $1.07-1.22$ & $<0.001$ \\
\hline Clinical adjustment ${ }^{b}$ & 1.05 & $0.98-1.13$ & 0.16 \\
\hline Age and clinical adjustment & 1.06 & $0.99-1.13$ & 0.08 \\
\hline
\end{tabular}

a For bilateral infiltrates in chest X-ray

b For modified early warning score

that: 1. All admitted patients, even with mild disease, have abnormal LUS at presentation; 2. For the majority of patients, the most common finding on LUS was patchy pleural thickening or patchy subpleural consolidations in at least one zone. 3. Increased LUS score is associated with worsening disease; 4 . In deteriorating patients, LUS pathology worsens mostly in the anterior lung segments and correlates with PEEP requirements. 5. Baseline LUS predicts death and/or clinical deterioration and may aid risk stratification and clinical decision making.

\section{Ultrasonographic features of COVID-19}

None of the patients had normal LUS, suggesting a possible role of LUS to rule out COVID-19 infection in symptomatic hospitalized patients. However, because less than $10 \%$ of symptomatic patients with COVID-19 infection are admitted to the hospital in Israel, these results are susceptible to selection bias. We believe that our results should serve as incentive to explore the role of LUS in ruling out COVID-19 infection in larger series, including asymptomatic as well as ambulatory patients. The most common findings were pleural thickening and subpleural consolidations whereas no homogenous diffuse B-lines were seen. Moreover, bedside echocardiography did not reveal findings suggestive of elevated left atrial pressure in the majority of patients. Such features correlate with previous high-resolution CT descriptions of patchy subpleural lung infiltrates in COVID-19 [25] and rules out the etiology of cardiogenic pulmonary edema [15].

\section{LUS findings in relation with disease severity}

LUS score in patients with severe disease were significantly higher compared with patients with mild or moderate disease. With worsening disease, more pleural thickening and subpleural consolidations were recorded. The relation between clinical severity and LUS findings is in line with previously published data using LUS and chest CT scores [26, 27], as well as with previously described patterns in swine (H1N1) and avian (H7N9) Influenza [28, 29]. Interestingly, the main contributor to the worsening LUS score was new, or greater, involvement of anterior segments, a finding that may be used clinically to warn from imminent deterioration. Furthermore, in patients who were mechanically ventilated during baseline LUS and later underwent a second examination due to clinical deterioration, LUS score and PEEP requirements were significantly correlated. Recent publications have shown that with respiratory distress from COVID-19, patients initially may retain relatively good lung compliance despite very poor oxygenation [30-32]. 
In these patients, CT exam will show limited groundglass infiltrates, signifying interstitial rather than alveolar edema [33, 34]. These patients have low response to PEEP, and tolerate larger tidal volumes $(7-8 \mathrm{~mL} / \mathrm{kg}$ ideal body weight). In some patients, the disease progressively develops into the "classic" type ARDS, with CT showing extensive consolidations associated with low lung compliance, and the need for higher PEEP, low tidal volume and early consideration of prone positioning $[35,36]$. When challenged by such a dynamic disease, a quick bedside imaging exam as LUS may become extremely helpful for distinguishing between these phenotypes, follow patients' clinical status and direct therapy accordingly, thus allowing adequate changes in respiratory support to higher PEEP, low tidal volume and early consideration of prone positioning in patients with increasing LUS scores and decreasing number of normal segments, suggesting rapidly decreasing compliance. Furthermore, our data show that in the final stages of clinical deterioration, even the anterior lung segments can become consolidated. This finding can predict a poor response to prone positioning [37].

\section{LUS as a predictive tool of clinical course and outcome}

Our data show that a higher LUS score, appearance of pleural thickening and pleural effusion predict the need for mechanical ventilation, mortality and the combination of both. Survival drops significantly with an LUS score above 18. This prediction is independent of chest $\mathrm{X}$-ray findings, making it a stand-alone superior alternative. For the composite outcome of need for invasive mechanical ventilation or death, the predictive ability of LUS score is even superior to that of chest X-ray and $\mathrm{O}_{2}$ saturation. This is in concordance with previously described evidence in patients with decompensated heart failure, in which semi-quantitative B-line assessment was shown to be a prognostic indicator of adverse outcomes and mortality [9]. Moreover, our results are in line with a publication regarding chest CT in COVID-19 patients, in which the total burden of lung involvement and anterior segment involvement at admission were associated with higher rates of adverse clinical composite endpoints of ICU admission, respiratory failure and shock [38]. The peripheral distribution of lung infiltrates in COVID-19 makes LUS a reliable imaging study, and can reduce the number of CT scans performed [17, 39], with their associated risks of infection spread, radiation exposure and the need to disinfect the CT room [22]. Moreover, transporting critical patients to $\mathrm{CT}$ is challenging and complex, while LUS can be easily performed at the bedside.

Our study identified patients without any pleural thickening or subpleural consolidations, who did not experience clinical deterioration, showing the ability of a straightforward baseline LUS to also predict a good clinical outcome and serve as a mean of triage, especially in case of widespread infection and emergency room overcrowding. It could also serve as an adjunct in hospitalized patients discharge decisions.

\section{Limitations}

First, our study is a single center study, which included only patients with COVID-19 who were hospitalized for at least $24 \mathrm{~h}$. The fact that only $\approx 7 \%$ of patients diagnosed with COVID-19 in Israel are admitted to the hospital, probably led to over-estimation of the severity of LUS in COVID-19. Fifteen patients (11.1\%) were excluded. Six of these patients were excluded due to "Do Not Resuscitate/Intubate" orders. These patients received only palliative care and died shortly after their admission. This fact may have created an opposite bias resulting in underestimation of LUS severity in patients with COVID-19 infection. Using phased-array transducers is acceptable when performing LUS, but its low frequency and high penetrance can compromise pleural evaluation. Nevertheless, placing the focus at the pleura level enabled reasonable assessment of the pleural line and subpleural consolidations. The fact that LUS measurements were calculated by the cardiologist caring for the patient may lead to over-estimation of the severity of LUS. Outcome analyses in our study should be interpreted with caution due to the small number of patients.

\section{Conclusions and clinical implications}

In patients with COVID-19, LUS rapidly identifies pulmonary involvement and provides risk stratification, including prediction of need for mechanical ventilation and mortality, above routine radiographic assessment. Its use may guide patients' management strategies, as well as resource allocation in case of surge capacity.

\section{Electronic supplementary material}

The online version of this article (https://doi.org/10.1007/s00134-020-06212-1) contains supplementary material, which is available to authorized users.

\section{Author details \\ ${ }^{1}$ Tel Aviv Sourasky Medical Center and Sackler School of Medicine, Tel Aviv University, Tel Aviv, Israel. ${ }^{2}$ Department of Cardiology, Tel Aviv Medical Center, Weizmann 6, 6423919 Tel Aviv, Israel.}

\section{Author contributions}

Conceptualization, methodology, formal analysis and original draft preparation: YS, YL, YT. All authors contributed to material preparation and data collection, commented on previous versions of the manuscript, read and approved the final manuscript.

\section{Funding}

None. 


\section{Data availability}

All data that support the findings of this study will be available from the corresponding author upon reasonable request.

\section{Code availability}

Not applicable.

\section{Compliance with ethical standards}

Conflicts of interest

The authors have no conflict of interest.

\section{Publisher's Note}

Springer Nature remains neutral with regard to jurisdictional claims in published maps and institutional affiliations.

Received: 11 June 2020 Accepted: 4 August 2020

Published online: 28 August 2020

\section{References}

1. Wang D, Hu B, Hu C, Zhu F, Liu X, Zhang J et al (2020) Clinical characteristics of 138 hospitalized patients with 2019 novel coronavirus-infected pneumonia in Wuhan China. JAMA 323(11):1061-1069

2. Chen N, Zhou M, Dong X, Qu J, Gong F, Han Y et al (2020) Epidemiological and clinical characteristics of 99 cases of 2019 novel coronavirus pneumonia in Wuhan, China: a descriptive study. Lancet 395(10223):507-513

3. Richardson S, Hirsch JS, Narasimhan M, Crawford JM, McGinn T, Davidson KW et al (2020) Presenting characteristics, comorbidities, and outcomes among 5700 patients hospitalized with COVID-19 in the New York City area. JAMA. https://doi.org/10.1001/jama.2020.6775

4. Zhou F, Yu T, Du R, Fan G, Liu Y, Liu Z et al (2020) Clinical course and risk factors for mortality of adult inpatients with COVID-19 in Wuhan, China: a retrospective cohort study. Lancet. https://doi.org/10.1016/S0140 $-6736(20) 30566-3$

5. Hu Z, Song C, Xu C, Jin G, Chen Y, Xu X et al (2020) Clinical characteristics of 24 asymptomatic infections with COVID-19 screened among close contacts in Nanjing China. Sci China Life Sci. https://doi.org/10.1007/ s11427-020-1661-4

6. Man MA, Dantes E, Domokos Hancu B, Bondor Cl, Ruscovan A, Parau A et al (2019) Correlation between Transthoracic Lung Ultrasound Score and HRCT Features in patients with interstitial lung diseases. J Clin Med 8(8):1199

7. Bouhemad B, Mongodi S, Via G, Rouquette I (2015) Ultrasound for "lung monitoring" of ventilated patients. Anesthesiology 122(2):437-447

8. Xirouchaki N, Kondili E, Prinianakis G, Malliotakis P, Georgopoulos D (2014) Impact of lung ultrasound on clinical decision making in critically ill patients. Intensive Care Med 40(1):57-65

9. Volpicelli G, Elbarbary M, Blaivas M, Lichtenstein DA, Mathis G, Kirkpatrick AW et al (2012) International evidence-based recommendations for point-of-care lung ultrasound. Intensive Care Med 38(4):577-591

10. Mayo PH, Copetti R, Feller-Kopman D, Mathis G, Maury E, Mongodi S et al (2019) Thoracic ultrasonography: a narrative review. Intensive Care Med. https://doi.org/10.1007/s00134-019-05725-8

11. Volpicelli G, Mayo P, Rovida S (2020) No title. Focus on ultrasound in intensive care. Intensive Care Med 46:1258-1260

12. Xirouchaki N, Magkanas E, Vaporidi K, Kondili E, Plataki M, Patrianakos A et al (2011) Lung ultrasound in critically ill patients: comparison with bedside chest radiography. Intensive Care Med 37(9):1488

13. Mayo PH, Beaulieu Y, Doelken P, Feller-Kopman D, Harrod C, Kaplan A et al (2009) American College of Chest Physicians/La Société de Réanimation de Langue Française statement on competence in critical care ultrasonography. Chest 135(4):1050-1060

14. Danish M, Agarwal A, Goyal P, Gupta D, Lal H, Prasad R et al (2019) Diagnostic performance of 6-point lung ultrasound in ICU patients: a comparison with chest X-ray and CT thorax. Turk J Anaesthesiol Reanim 47(4):307
15. Lichtenstein DA (2015) BLUE-protocol and FALLS-protocol: two applications of lung ultrasound in the critically ill. Chest 147(6):1659-1670

16. Corradi F, Brusasco C, Pelosi P (2014) Chest ultrasound in acute respiratory distress syndrome. Curr Opin Crit Care 20(1):98-103

17. Vetrugno L, Bove T, Orso D, Barbariol F, Bassi F, Boero E et al (2020) Our Italian experience using lung ultrasound for identification, grading and serial follow-up of severity of lung involvement for management of patients with COVID-19. Echocardiography 37(4):625-627

18. Mayo P, Arntfield R, Balik M, Kory P, Mathis G, Schmidt G et al (2017) The ICM research agenda on critical care ultrasonography. Intensive Care Med 43(9):1257-1269

19. Szekely Y, Lichter Y, Taieb P, Banai A, Hochstadt A, Merdler I et al (2020) The spectrum of cardiac manifestations in Coronavirus Disease 2019 (COVID19)-a systematic echocardiographic study. Circulation 142:342-353

20. Lambden S, Laterre PF, Levy MM, Francois B (2019) The SOFA scoredevelopment, utility and challenges of accurate assessment in clinical trials. Crit Care 23(1):1-9

21. Liao X, Wang B, Kang Y (2020) Novel coronavirus infection during the 2019-2020 epidemic: preparing intensive care units — the experience in Sichuan Province China. Intensive Care Med 46(2):357-360

22. Kirkpatrick JN, Mitchell C, Taub C, Kort S, Hung J, Swaminathan M (2020) ASE statement on protection of patients and echocardiography service providers during the 2019 novel coronavirus outbreak. J Am Coll Cardiol. https://doi.org/10.1016/j.echo.2020.04.001

23. Volpicelli G, Lamorte A, Villén T (2020) What's new in lung ultrasound during the COVID-19 pandemic. Intensive Care Med. https://doi.org/10.1007/ s00134-020-06048-9

24. Peng Q, Wang X, Zhang L, Chinese Critical Care Ultrasound Study Group (2020) Findings of lung ultrasonography of novel corona virus pneumonia during the 2019-2020 epidemic. Intensive Care Med. https://doi. org/10.1007/s00134-020-05996-6

25. Jajodia A, Ebner L, Heidinger B, Prosch H (2019) Imaging in corona virus disease 2019 (COVID-19)_A scoping review. Eur J Radiol Open 2020:100237

26. Li K, Wu J, Wu F, Guo D, Chen L, Fang Z et al (2020) The clinical and chest CT features associated with severe and critical COVID-19 pneumonia. Invest Radiol 55(6):327-331

27. Zieleskiewicz L, Markarian T, Lopez A, Taquet C, Mohammedi N, Boucekine M et al (2020) Comparative study of lung ultrasound and chest computed tomography scan in the assessment of severity of confirmed COVID-19 pneumonia. Intensive Care Med. https://doi. org/10.1007/s00134-020-06186-0

28. Peris A, Zagli G, Barbani F, Tutino L, Biondi S, Di Valvasone S et al (2010) The value of lung ultrasound monitoring in $\mathrm{H} 1 \mathrm{~N} 1$ acute respiratory distress syndrome. Anaesthesia 65(3):294-297

29. Tsai NW, Ngai CW, Mok KL, Tsung JW (2014) Lung ultrasound imaging in avian influenza A (H7N9) respiratory failure. Critic Ultrason J 6(1):6

30. Grasselli G, Zangrillo A, Zanella A, Antonelli M, Cabrini L, Castelli A et al (2020) Baseline characteristics and outcomes of 1591 patients infected with SARS-CoV-2 admitted to ICUs of the Lombardy region Italy. JAMA 323(16):1574-1581

31. Arentz M, Yim E, Klaff L, Lokhandwala S, Riedo FX, Chong M et al (2020) Characteristics and outcomes of 21 critically ill patients with COVID-19 in Washington State. JAMA 323(16):1612-1614

32. Gattinoni L, Chiumello D, Caironi P, Busana M, Romitti F, Brazzi L et al (2020) COVID-19 pneumonia: different respiratory treatments for different phenotypes? Intensive Care Med 46:1099-1102

33. Bernheim A, Mei X, Huang M, Yang Y, Fayad ZA, Zhang N et al (2020) Chest CT findings in coronavirus disease-19 (COVID-19): relationship to duration of infection. Radiology 295:200463

34. Pan F, Ye T, Sun P, Gui S, Liang B, Li L et al (2019) Time course of lung changes on chest CT during recovery from 2019 novel coronavirus (COVID-19) pneumonia. Radiology 2020:200370

35. Amato MBP, Barbas CSV, Medeiros DM, Magaldi RB, Schettino GP, LorenziFilho $G$ et al (1998) Effect of a protective-ventilation strategy on mortality in the acute respiratory distress syndrome. N Engl J Med 338(6):347-354

36. Guérin C, Reignier J, Richard J, Beuret P, Gacouin A, Boulain T et al (2013) Prone positioning in severe acute respiratory distress syndrome. N Engl J Med 368(23):2159-2168 
37. Prat G, Guinard S, Bizien N, Nowak E, Tonnelier J, Alavi Z et al (2016) Can lung ultrasonography predict prone positioning response in acute respiratory distress syndrome patients? J Crit Care 32:36-41

38. Yu Q, Wang Y, Huang S, Liu S, Zhou Z, Zhang S et al (2020) Multicenter cohort study demonstrates more consolidation in upper lungs on initia CT increases the risk of adverse clinical outcome in COVID-19 patients. Theranostics 10(12):5641
39. Mongodi S, Orlando A, Arisi E, Tavazzi G, Santangelo E, Caneva L et al (2020) Lung ultrasound in patients with acute respiratory failure reduces conventional imaging and health care provider exposure to COVID-19. Ultrasound Med Biol 46:2090-2093 